\title{
Dynamics of an Earthquake under Magma Thrust Strength
}

\author{
L. Y. Kagho, M. W. Dongmo, and F. B. Pelap \\ Laboratoire de Mécanique et de Modélisation des Systèmes Physiques (L2MSP), Faculté des Sciences, Université de Dschang, \\ BP 69, Dschang, Cameroon \\ Correspondence should be addressed to F. B. Pelap; fbpelap@yahoo.fr
}

Received 22 November 2014; Accepted 9 February 2015

Academic Editor: Alejandro Ramírez-Rojas

Copyright (C) 2015 L. Y. Kagho et al. This is an open access article distributed under the Creative Commons Attribution License, which permits unrestricted use, distribution, and reproduction in any medium, provided the original work is properly cited.

This paper deals with the study of the behaviour of a one spring-block model subjected to the strengths due to the motion of the tectonic plates and the upflow of magma during volcanism. Using the direct integration method, we show that the sound velocity decreases (or increases) with the amplitude of the block's oscillation when the external frequency is zero (or not). It appears that this amplitude grows with the amplitude of the magma thrust strength. For the resonance case, where this external frequency equals the pseudofrequency of the block's oscillation, we establish that the slip which occurs during the periodic movement of a block is a stick-slip motion instead of a creep motion as usually known; therefore, the transition does not occur. We also obtain that the event probability decreases with the amplitude of the magma thrust strength while the power of the earthquake increases with it.

\section{Introduction}

Many research works related to earthquake dynamics were focused on the study of a single spring-block model [1-3]. This single block model is deduced from the simplification of models of several blocks [4-8]. In these models, the motion of the fault is only stimulated by the strength due to the motion of the tectonic plates. As far as the one spring-block model is concerned, few studies have shown the appearance of chaotic behavior within the system when its motion is unidirectional [3] or bidirectional [2]. Moreover, Vasconcelos has investigated the phase transition in this single-block model and demonstrated that when the friction characteristic velocity is greater than 0.5 , the block displacement vanishes after a slip event and the transition occurs [1]. In this study, the only force that provokes the rupture of the fault is induced by the movement of tectonic plates. One feature of our work is to examine this transition in the presence of a supplementary action referred to as the magma thrust strength.

In this paper, we consider a modified $1 \mathrm{D}$ spring-block model subjected to the strength due to the movement of the tectonic plates during subduction movement and to the magma thrust strength that appears during a volcanic activity. Therefore, we plan to present the origin and investigate the effects of this new force on the system and thereby contribute to the better understanding of earthquake dynamics.
This paper is outlined as follows. In Section 2, we present the earthquake model under consideration and determine the equation of motion. Section 3 is devoted to the analytical analysis of the motion equation exploiting the direct integration method. These investigations are done for two cases which deal with the closeness (or not) between the frequency of the magma and the fundamental frequency of the spring block. In Section 4, the magnitude and the event probability of an earthquake are examined. The last section is devoted to discussions and concluding remarks.

\section{Earthquake Model and Equation of Motion}

The mechanical model under consideration (Figure 1) consists of a block of mass $m$ attached to a spring of stiffness $k_{p}$ that moves the upper line with a constant velocity $V$ which represents the speed of the tectonic plate. This block rests on a rough surface and is connected by a harmonic spring of stiffness $k_{c}$ which stands for the elasticity of rocks separating the block of mass $m$ and the loading point of the strength $F(t)$ that represents the magma thrust.

It is well known that the subduction movement is responsible of many earthquakes (tectonic event). However, many events (volcano-tremor, volcano-tectonic) are often recorded around active volcanoes. It is also known that volcanotectonic events are due to fault's sliding under the volcano 


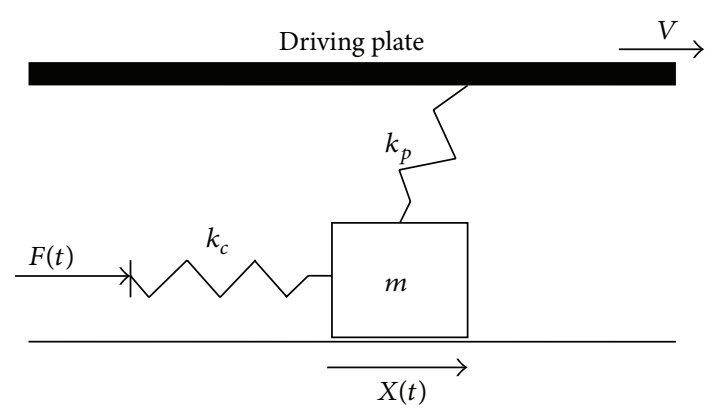

FIGURE 1: Spring-block model of earthquakes under consideration. Forces that intervene in this system are, respectively, the strength $F(t)$ due to the magma upflow, the elastic force $f_{1}(X)=-k_{c} X$ due to the contraction or dilatation of the spring of stiffness $k_{c}$, the external force $f_{2}(X)=-k_{p}(X-V t)$ due to the motion of the driving plate, and the frictional force $f(d X / d t)$ that deals with the asperities between the lips.

activity. The interior of a volcano is modeled by many authors [9-13] who showed that active volcano is one of the main sources of earthquakes particularly when the plates are initially loaded (Figure 2). They presented the interior of a volcano consisting of a shallow magma reservoir fed by a deeper source and a cylindrical-shaped conduit that transports the fluid upwards and may be connected to a network of other conduits [9]. Generally, the reservoir is considered to behave elastically under variable stress conditions imposed by fluid accumulation or outflow. On the other hand, the cylindrical conduit is believed to exhibit more complicated viscous behavior so that a high driving fluid pressure from below results in deformations transmitted periodically as expansions or contractions of its walls $[11,13]$. After a relative long time, these periodic deformations induced a periodic strength that expanded to the rocks nearby during the motion of the magma. Hereafter, we refer to this force as the magma thrust strength $F(t)$ defined by

$$
F(t)=F_{M} \cos \left(\omega_{e} t\right),
$$

where $F_{M}$ represents the amplitude of the force and depends on the magma characteristics: magma viscosity $\left(\eta_{1}\right)$, rock viscosity $\left(\eta_{2}\right)$, the radius of the magmatic room $(R)$, gravity $(g)$, that is, $F_{M}=F\left(\eta_{1}, \eta_{2}, R, g\right)$, and $\omega_{e}$ which is the external frequency. Hereafter, $F(t)$ could be referred to as the external force acting on the block of mass $m$. During the investigation of the dynamics of our system, we assume that the fracturing zone where the magma outbreaks is orthogonal to the fault [14]. Then, the forces created by the thrust of the magma on the earth crust are parallel to the fault during the volcanic emission.

Initially $(t=0)$, we consider that the system is at rest, and the elastic energy accumulated in the horizontal spring $k_{c}$ is due to the force $f_{1}(X)=-k_{c} X$ that yields by the contraction or dilation of this spring. The motion of the driving plate also participates in the movement of the block by exerting an external force of the form $f_{2}(X)=-k_{p}(X-V t)$ [1]. The asperities which exist between the lips generate the frictional force $f(d X / d t)$ which is opposed to the motion of the block.
During volcano emission, the system is also subjected to a force $F(t)$ due to the upflow of the magma. Therefore, the system of Figure 1 could be described by the upcoming equation in terms of real variables:

$$
m \frac{d^{2} X}{d t^{2}}=-k_{p}(X-V t)-k_{c} X-f\left(\frac{d X}{d t}\right)+F(t) .
$$

It should be stressed that there exist several models of frictional force $[1,8,15,16]$. If we suppose that the speeds are low during the sliding, then we can consider the Vasconcelos frictional model [1] defined by

$$
f\left(\frac{d X}{d t}\right)=F_{0} \Phi\left(\frac{1}{v_{f}} \frac{d X}{d t}\right)
$$

wherein $F_{0}$ is the amplitude of frictional force, $v_{f}$ stands for the characteristic velocity of friction, and $\Phi(x)$ represents a continuous function for $x \geq 0$ that vanishes for large values of $x$ and satisfies the following conditions:

$$
\Phi(0)=1, \quad \Phi^{\prime}(0)=-1
$$

in which the prime denotes differentiation with respect to the argument. The second condition in (4) expresses the velocityweakening effects of the friction and implies that $f(d X / d t)$ will be a decreasing function of $d X / d t$.

Substitution of the mechanical actions (1) and (3) into (2) leads to

$$
\begin{aligned}
m \frac{d^{2} X}{d t^{2}}= & -k_{p}(X-V t)-k_{c} X-F_{0} \Phi\left(\frac{1}{v_{f}} \frac{d X}{d t}\right) \\
& +F_{M} \cos \left(\omega_{e} t\right) .
\end{aligned}
$$

We transform this equation into dimensionless form by defining new variables $U=k_{p} X / F_{0}$ and $\tau=t \sqrt{k_{p} / m}=\omega_{p} t$ so that (5) becomes as follows:

$$
\frac{d^{2} U}{d \tau^{2}}=-\left(1+l^{2}\right) U-\Phi\left(\frac{1}{\nu_{f}} \frac{d U}{d \tau}\right)+v \tau+F \cos (\omega \tau),
$$

where $l=\sqrt{k_{c} / k_{p}}$ defines the sound velocity, $v_{f}=V_{f} / V_{0}$ is the dimensionless characteristic velocity of friction, $v=$ $V / V_{0}$ designates the dimensionless driven velocity, $V_{0}=$ $F_{0} / \sqrt{k_{p} m}$ is the maximum velocity attained by the block that experiences friction as it moves, $\omega=\omega_{e} / \omega_{p}$ defines the dimensionless external frequency, and $F=F_{M} / F_{0}$ stands for the dimensionless amplitude of the external force.

At this level, if we assume that $(d U(\tau) / d \tau) \ll v_{f}$, then the function $\Phi\left(\left(1 / \nu_{f}\right)(d U / d \tau)\right)$ can be developed in the linear approximation in terms of Taylor series according to Langer and Tang [17]. Therefore, (6) takes the following form:

$$
\frac{d^{2} U}{d \tau^{2}}=-\left(1+l^{2}\right) U-\left(1-\frac{1}{\nu_{f}} \frac{d U}{d \tau}\right)+v \tau+F \cos (\omega \tau) \text {. }
$$




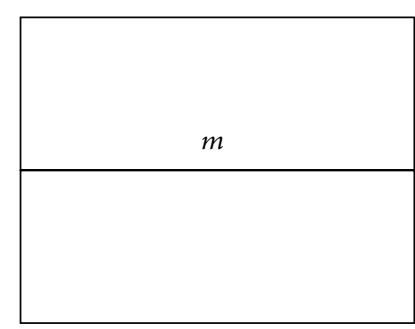

(a) Initial position of the block

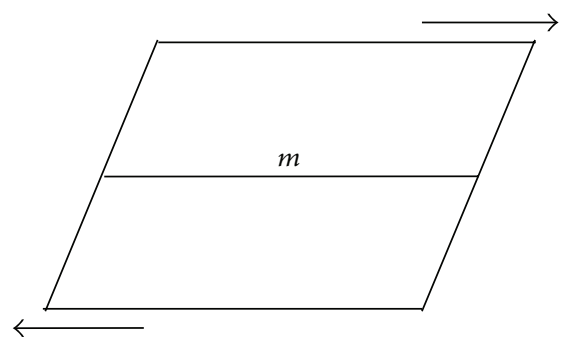

(b) Load of the block under the driving plate action

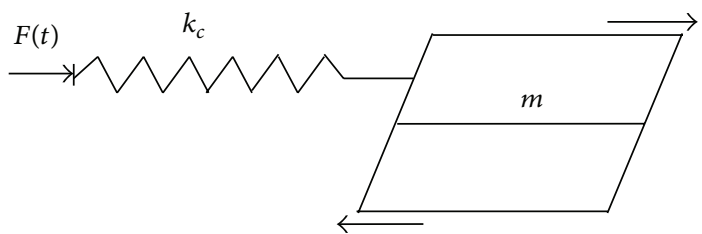

(c) Before the action $F(t)$

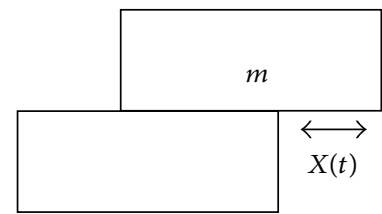

(d) After the cumulative action of

$F(t)$ and the driving plate

Figure 2: Action on the magma thrust strength on the block of mass.

Now, let us redefine the origin of displacement so as to eliminate the unit constant that would otherwise appear on the right-hand side of (7). Hence, the motion equation is put in the simplest form:

$$
\frac{d^{2} U}{d \tau^{2}}-2 \alpha \frac{d U}{d \tau}+\omega_{0}^{2} U=v \tau+F \cos (\omega \tau),
$$

where $\alpha=1 / 2 v_{f}$ is the friction coefficient and $\omega_{0}=\sqrt{1+l^{2}}$ defines the natural frequency. Equation (8) governs the slip of the block subjected to both the movement of the driving plate and the action of the magma thrust strength. It satisfies the initial conditions as below:

$$
U(0)=\frac{d U(0)}{d \tau}=0 .
$$

Expression (8) is the equation of motion that characterizes the movement of the block. If $\omega_{0}=1$ (i.e., when the sound velocity is zero) and $F=0$, we recover the Vasconcelos results [1]. In the upcoming sections, we carry out the analytical analysis of (8).

\section{Analytical Analysis}

In this section, we intend to solve (8) and examine the properties of its solutions. Observation of this equation shows that one can distinguish two subcases that deal with the sign of the discriminant $\alpha^{2}-\omega_{0}^{2}$ of the characteristic equation associated with (8). Hereafter, we will investigate separately the behavior of the system in each case.

3.1. Case Where $\alpha \geq \omega_{0}$. This case corresponds to the physical situation in which the frictional coefficient is greater than the natural frequency of the block. Mathematically, the general solution of (8) is sought under the form:

$$
U(\tau)=U^{*}(\tau)+\bar{U}(\tau)
$$

in which $U^{*}(\tau)$ is the solution of the homogeneous equation and $\bar{U}(\tau)$ is a particular solution of the entire equation (8). Computations show that the solution $U^{*}(\tau)$ has the explicit form:

$$
U^{*}(t)=\exp (\alpha \tau)\left[A_{1} \cosh (\widetilde{\omega} \tau)+B_{1} \sinh (\widetilde{\omega} \tau)\right]
$$

wherein $A_{1}$ and $B_{1}$ are integration constants to be determined by using the initial conditions.

The last part of solution (10) can be put under the form:

$$
\bar{U}(t)=a t+d+b \cos (\omega \tau)+c \sin (\omega \tau),
$$

where $a, d, b$, and $c$ are constants to be determined. While exploiting (12) and (8), we find that

$$
\begin{gathered}
a=\frac{\nu}{\omega_{0}^{2}}, \quad d=\frac{2 \alpha \nu}{\omega_{0}^{4}}, \\
b=\frac{F\left(\omega_{0}^{2}-\omega^{2}\right)}{\left(\omega_{0}^{2}-\omega^{2}\right)^{2}+4 \alpha^{2} \omega^{2}}, \\
c=-\frac{2 \alpha \omega F}{\left(\omega_{0}^{2}-\omega^{2}\right)^{2}+4 \alpha^{2} \omega^{2}} .
\end{gathered}
$$

Hence, we substitute (11) and (12) into (10) and use the initial conditions (9). Therefore, the displacement $U(\tau)$, solution of (8), is written as

$$
\begin{aligned}
U(\tau)= & \exp (\alpha \tau)\left[A_{1} \cosh (\widetilde{\omega} \tau)+B_{1} \sinh (\widetilde{\omega} \tau)\right] \\
& +a t+d+b \cos (\omega \tau)+c \sin (\omega \tau)
\end{aligned}
$$



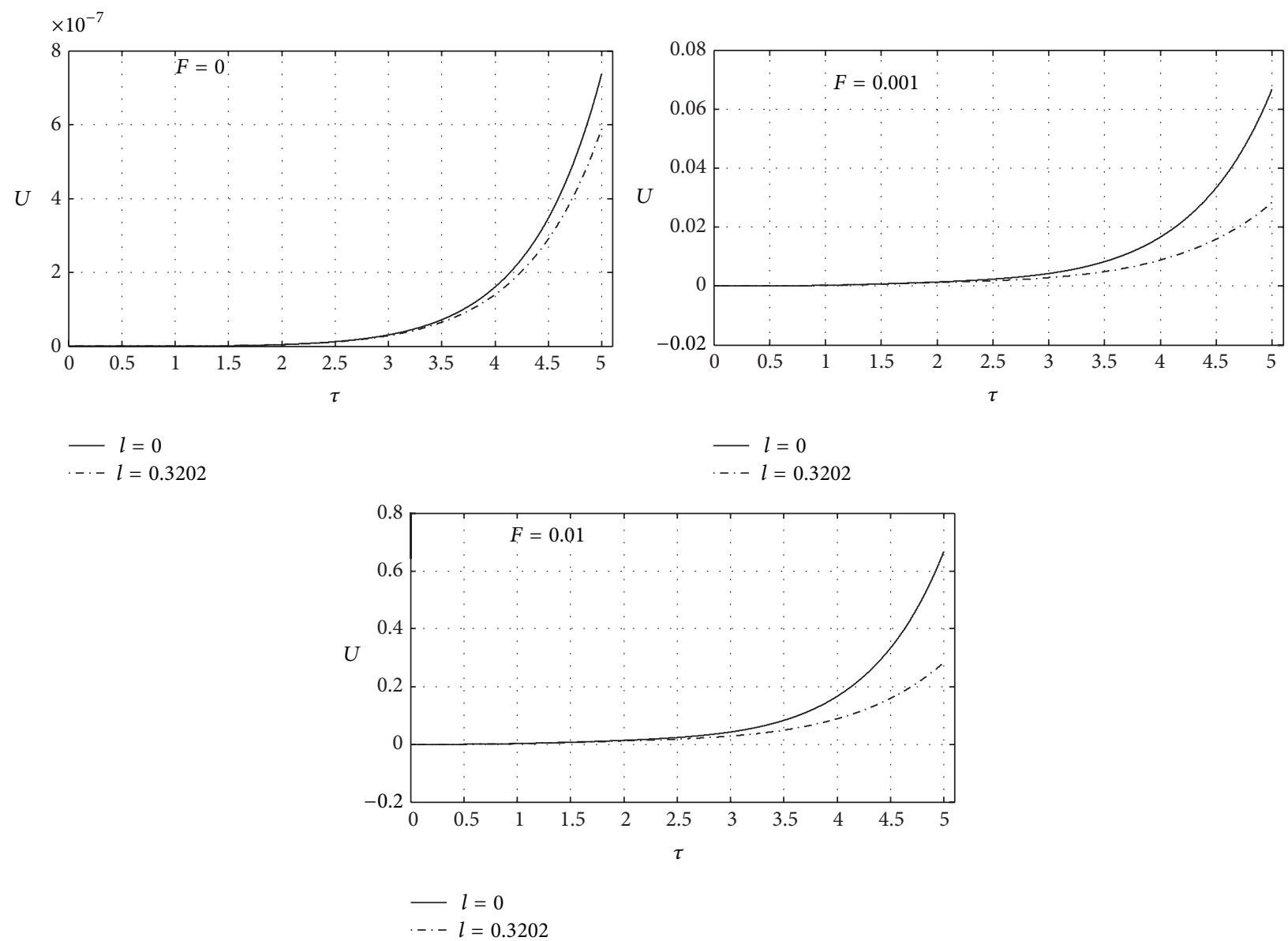

FIGURE 3: Influence of the magma thrust strength and the sound velocity on the slip for the parameters $\alpha=1.06$ and $\omega=1.5$.

with

$$
\begin{gathered}
A_{1}=-\left[\left(\frac{2 \alpha \nu}{\omega_{0}^{4}}\right)+\frac{F\left(\omega_{0}^{2}-\omega^{2}\right)}{\left(\omega_{0}^{2}-\omega^{2}\right)+4 \alpha^{2} \omega^{2}}\right], \\
B_{1}=\frac{1}{\widetilde{\omega}}\left[\nu\left(\frac{2 \alpha^{2}}{\omega_{0}^{4}}-\frac{1}{\omega_{0}^{2}}\right)+\frac{F \alpha\left(\omega_{0}^{2}+\omega^{2}\right)}{\left(\omega_{0}^{2}-\omega^{2}\right)+4 \alpha^{2} \omega^{2}}\right], \\
\widetilde{\omega}=\sqrt{\alpha^{2}-\omega_{0}^{2}} .
\end{gathered}
$$

For this case, where $\alpha \geq \omega_{0}$, we use solution (14) and examine the impact of the amplitude of the magma thrust strength $F$ and that of the sound velocity $l$ on the behavior the block. Numerical results are plotted in Figure 3.

Figure 3 shows that the slip increases with the time and the amplitude of the external force but decreases with the growth of the sound velocity. Curves of Figure 3 which deal with the parameters $l=0$ and $F=0$ correspond to the results established by Vasconcelos [1]. On these curves, the slip is very low (of $10^{-7}$ order) compared to the case where the magma strength is not zero. The decay of the slip with the growth of the sound velocity explains the role played by the horizontal spring of our model. Moreover, when the horizontal spring is removed, the external force acts directly on the block. Then, the slip which occurs is greater than the results obtained in the presence of the horizontal spring.

3.2. Case Where $\alpha<\omega_{0}$. In this subsection, we focus our attention in the physical situation where the frictional coefficient is less than the natural frequency of the block. Here, the displacement of the block $U(\tau)$, solution of the motion equation (8), is given by

$$
\begin{aligned}
U(\tau)= & \exp (\alpha \tau)\left[A_{1} \cos (\tilde{\omega} \tau)+B_{1} \sin (\tilde{\omega} \tau)\right] \\
& +a t+d+b \cos (\omega \tau)+c \sin (\omega \tau)
\end{aligned}
$$

with $\widetilde{\omega}=\sqrt{\omega_{0}^{2}-\alpha^{2}}$.

In order to measure the effects of the magma thrust strength on the dynamics of the system, it is necessary to investigate the temporal evolution of the displacement $U(\tau)$ when those parameters vary.

3.2.1. Temporal Evolution of the Displacement. Here, we examine the impact of the amplitude and the frequency of the external force as well as the effects of the sound velocity on the dynamics of the system. We believe necessary to examine the two different cases dealing with $\omega=0$ and $\omega \neq 0$, respectively. 

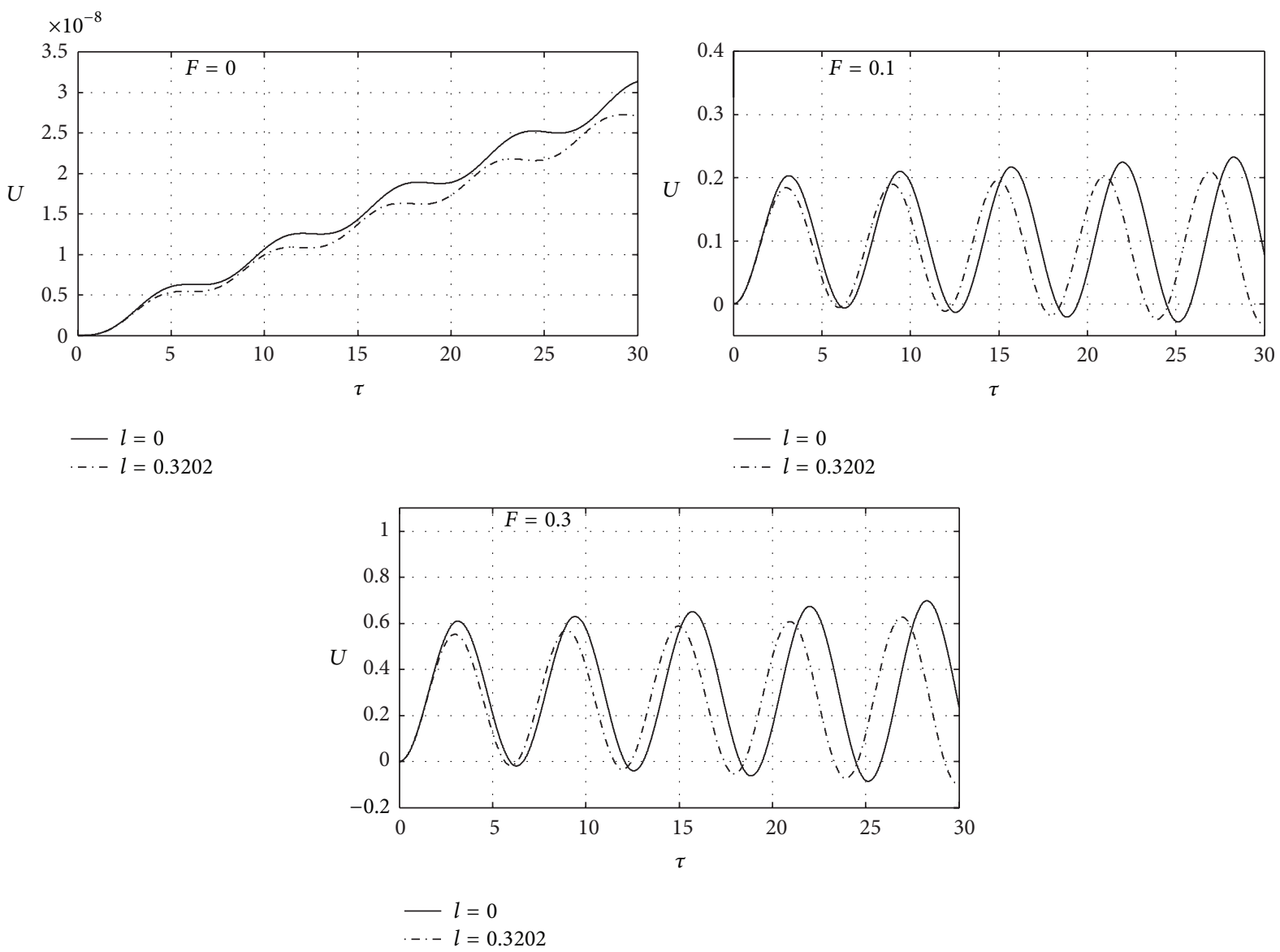

FIGURE 4: Temporal evolution of the block displacement when the amplitude of the magma thrust strength and the sound velocity vary for $\alpha=0.01$ and $\omega=0$.

When $\omega=0$, the external force exerted by the magma on the block becomes constant. Figure 4 shows that the displacement of the block is oscillatory. It also appears from these graphs that the amplitude of this displacement increases with the growth of the amplitude of the external force but decays while the sound velocity increases.

When $\omega \neq 0$, one notes from Figure 4 that the displacement of the block is still oscillatory and its amplitude increases with the growth of the magma thrust strength amplitude. These curves also exhibit the increment of the displacement with the sound velocity for nonzero values of $F$.

Figures 4 and 5 enable us to appreciate the effects of the external frequency on the dynamics of the system. One notices that, for $\omega=0$, the displacement of the block presents a simple oscillatory behavior while its profile becomes complex for nonnull values of the external frequency.

\subsubsection{Displacement of the Block at the End of the Earthquake.} At the end of the slip, the block occupies an undefined position that can be identical or not to his initial position. The length between the final and initial positions of the block depends on the forces which have generated the slip. Vasconcelos [1] showed that when the displacement of the block is provoked only by the movement of the tectonic plates, the block exhibits a creep motion since its initial and final positions after the slip are identical. Therefore, its displacement is null when $v \rightarrow 0$.

In this subsection, we wish to evaluate this position when the block is subjected to cumulative actions created by the motion of the tectonic plates and the magma upflow. Indeed, when the block ceases to move, it occupies a position whose determination requires the knowledge of the time $\tau_{0}$ at which the block stopped to slip. Concretely, when the block stops, its speed vanishes. Then, the corresponding time $\tau_{0}$ is the solution of the following equation:

$$
\begin{aligned}
& {\left[\left(A_{1} \alpha+\widetilde{\omega} B_{1}\right) \cos \left(\widetilde{\omega} \tau_{0}\right)+\left(B_{1} \alpha-\widetilde{\omega} A_{1}\right) \sin \widetilde{\omega} \tau_{0}\right] \exp \left(\alpha \tau_{0}\right)} \\
& \quad+c \omega \cos \left(\omega \tau_{0}\right)-b \omega \sin \left(\omega \tau_{0}\right)+\frac{\nu}{\omega_{0}^{2}}=0 .
\end{aligned}
$$

Now, we plan to solve analytically this equation. For simplification matters, we consider the particular case where the pseudofrequency of the block equals the frequency of the magma thrust strength (i.e., $\widetilde{\omega}=\omega$ ). Therefore, expression (17) is reduced to

$$
\begin{aligned}
& {\left[\left(A_{1} \alpha+\widetilde{\omega} B_{1}\right) \exp \left(\alpha \tau_{0}\right)+\widetilde{\omega} c\right] \cos \left(\widetilde{\omega} \tau_{0}\right)} \\
& \quad+\left[\left(B_{1} \alpha-\widetilde{\omega} A_{1}\right) \exp \left(\alpha \tau_{0}\right)-\widetilde{\omega} b\right] \sin \left(\widetilde{\omega} \tau_{0}\right)+\frac{\nu}{\omega_{0}^{2}}=0 .
\end{aligned}
$$



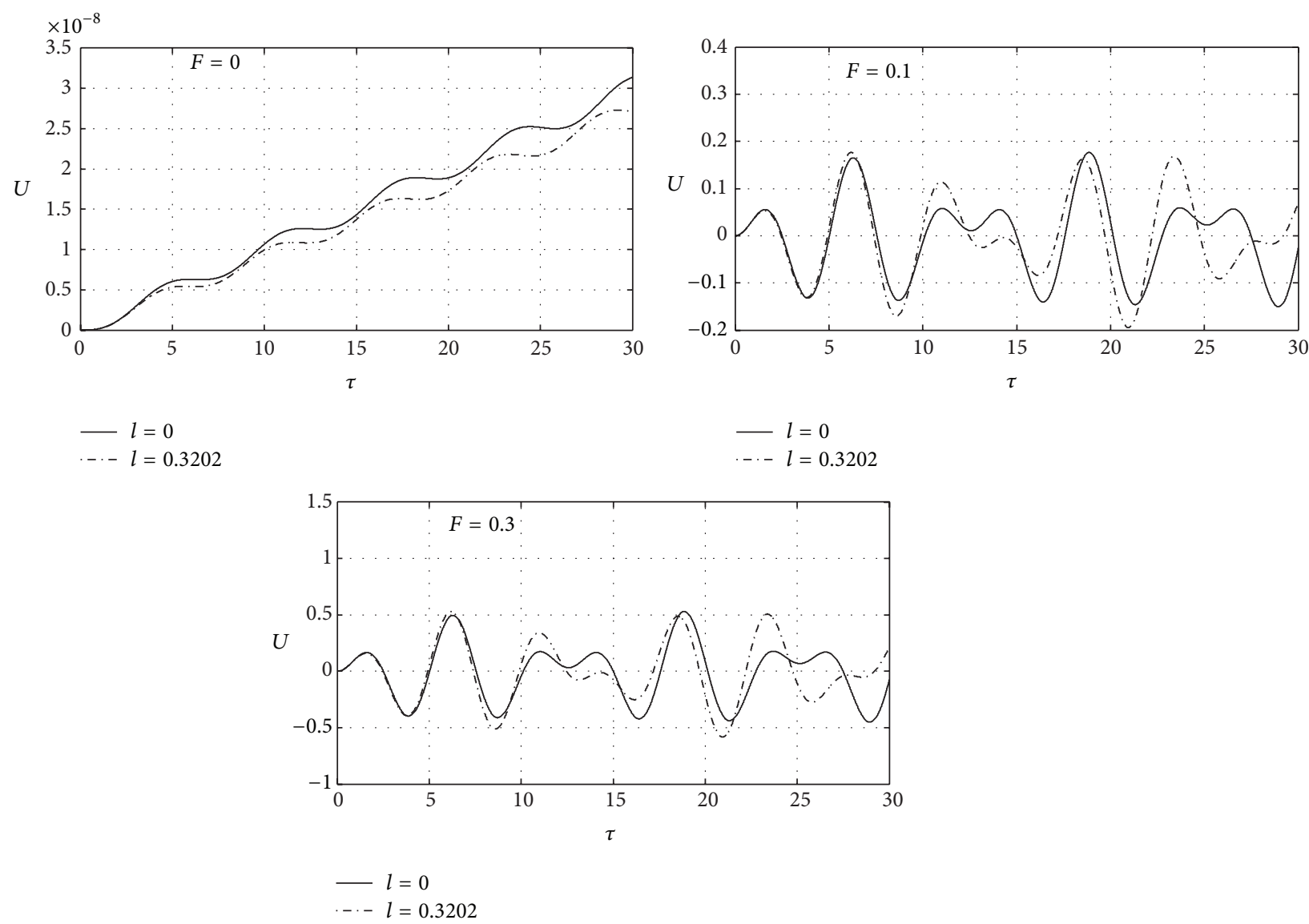

Figure 5: Time evolution of the block displacement for varying values of the amplitude of the magma thrust strength and the sound velocity for $\alpha=0.01$ and $\omega=1.5$.

Making use of relations (18) and (16) to find the final position of the block, we obtain that it depends on the duration of the slip event $\tau_{0}$ :

$$
\begin{aligned}
\Delta= & \left(B_{1} \exp \left(\alpha \tau_{0}\right)+c\right) \xi\left(\tau_{0}\right) \\
& +\left(A_{1} \exp \left(\alpha \tau_{0}\right)+b\right) \sqrt{1-\xi^{2}\left(\tau_{0}\right)}+\nu\left(\frac{\tau_{0}}{\omega_{0}^{2}}+\frac{2 \alpha}{\omega_{0}^{4}}\right),
\end{aligned}
$$

wherein $\xi\left(\tau_{0}\right)=\left(\nu \zeta_{2}\left(\tau_{0}\right)-\zeta_{1}\left(\tau_{0}\right) \sqrt{\omega_{0}^{2} \zeta_{3}\left(\tau_{0}\right)-\nu^{2}}\right) / \zeta_{3}\left(\tau_{0}\right)$ $\zeta_{1}\left(\tau_{0}\right)=\left(A_{2} \exp \left(\alpha \tau_{0}\right)+\widetilde{\omega} c\right), \zeta_{2}\left(\tau_{0}\right)=B_{2} \exp \left(\alpha \tau_{0}\right)-\widetilde{\omega} b$, $\zeta_{3}\left(\tau_{0}\right)=\omega_{0}^{2}\left(\zeta_{1}^{2}\left(\tau_{0}\right)+\zeta_{2}^{2}\left(\tau_{0}\right)\right), B_{2}=\alpha A_{1}+\widetilde{\omega} B_{1}, A_{2}=B A_{1}-\widetilde{\omega} A_{1}$.

While exploiting expression (19) in the limit case, where $v \rightarrow 0$, we note that the position of the block at the end of the earthquake is not null $(\Delta \neq 0)$. Physically, this result means that the slip which occurs is a stick-slip motion. Consequently, the fault remains seismologically active. It is not useless to remember that when the motion of the block is only provoked by the subduction movement, its displacement at the end of the slip is zero $(\Delta=0)$ for $v \rightarrow 0$ and the block experiences a creep motion. In this case, the fault is said to be seismologically inactive [1].
These results present the great role played by the magma thrust strength $F$ in the dynamical behavior of the earthquake. First of all, Vasconcelos [1] demonstrated that the aperiodic movement generates a stick-slip motion while the periodic oscillation induces a creep motion, and the transition occurs. In the present study, Figure 6 shows that when $F=0$, the position occupied by the block at the end of earthquake is zero $(\Delta=0)$. Therefore, the movement created by the oscillatory behavior of the block is a stick-slip. On the other hand, it appears from this figure that when $F \neq 0$, the position occupied by the block at the end of the earthquake is nonnull $(\Delta \neq 0)$. Then, the block experiences a stickslip motion. These results show that when the rupture of a fault occurs, the motion generated is a stick-slip irrespective of the nature of the movement (periodic or aperiodic); consequently, there is no transition. In comparison to the results of [1], the transformation of the creep to stick-slip occurs because of the magma thrust strength action. Equally, we note from Figure 6 that $\Delta$ increases lineally with $F$. It arises from this result that the more the action of this external load is considerable, the more the probability of the transition occurrence is weak. Thus, if an external force amplifies the movement of the tectonic plate to destabilise a fault, then phase transition will not be observed. In what follows, 


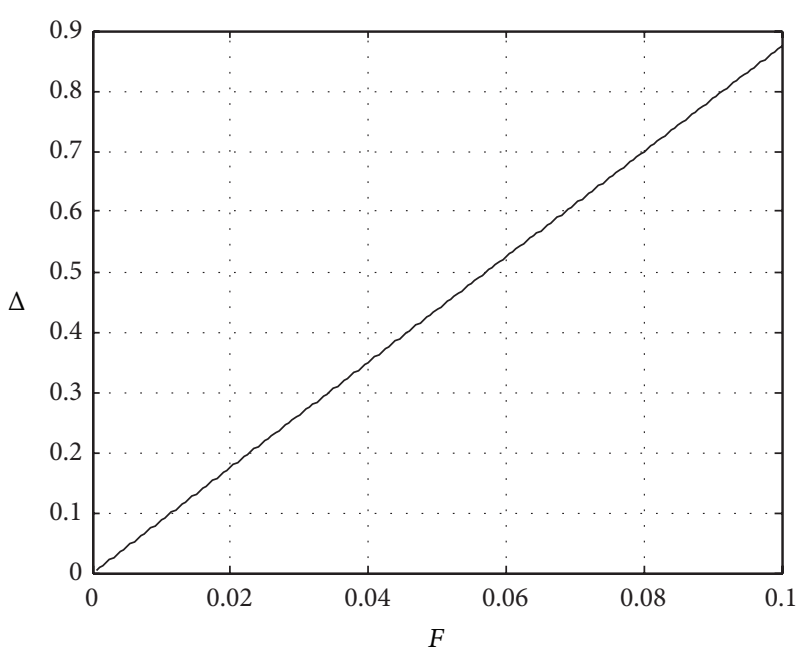

Figure 6: Position of the block at the end of the slip versus the amplitude of the external force for the parameters $\alpha=0.01, \omega_{0}=2$, and $\tau_{0}=30$.

the effects of $F$ on the power and the probability of an event are investigated.

\section{Magnitude of the Earthquake and Probability of an Event}

In this section, we evaluate the power of the earthquake (i.e., the magnitude) and the probability of an event when the system under consideration is defined by Figure 1. As the magnitude is concerned, various expressions have been proposed within which some consider the physical properties of the rocks [18-21] and others take into account the parameters characterizing the seismic waves $[22,23]$. Hereafter, we work in the former case with the magnitude expression given by

$$
M=\frac{2}{3}\left[\log _{10}\left(\frac{M_{0}}{1 N \cdot m}\right)-9.1\right]
$$

and the probability of an event defined in Gutenberg and Richter [24] by

$$
W=10^{\left(e-h \times \log M_{0}\right)}
$$

In these quantities, $M_{0}$ represents the seismic moment introduced by $[25,26]: M_{0}=\mu \cdot S \cdot \Delta$ in which $\mu$ is the rocks rigidity, $S$ characterizes the surface broken during the earthquake, and $\Delta$ designates the displacement of the block during the motion or its position after the seism. Since the broken surface and the rock rigidity are unknown, we consider the explicit forms of expressions (20) and (21) defined in [27]:

$$
\begin{gathered}
M=\frac{2}{3}\left[\log _{10}\left(K \cdot \Delta^{3}\right)-9.1\right], \\
W=10^{e-h \times \log \left(K \cdot \Delta^{3}\right)}
\end{gathered}
$$

in which $e$ and $h$ are arbitrary constants and $K$ is a constant which can be determined by exploiting the experimental

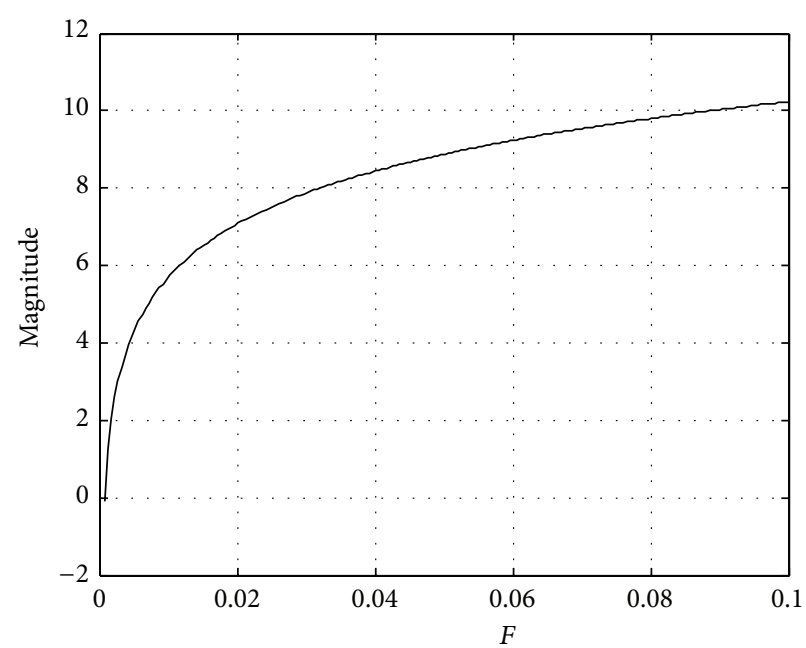

FIGURE 7: Magnitude of the seism as a function of the amplitude of the external force for the parameters $\alpha=0.01, \omega_{0}=2, \tau_{0}=30$, and $k=1.2589254 \times 10^{16}$.

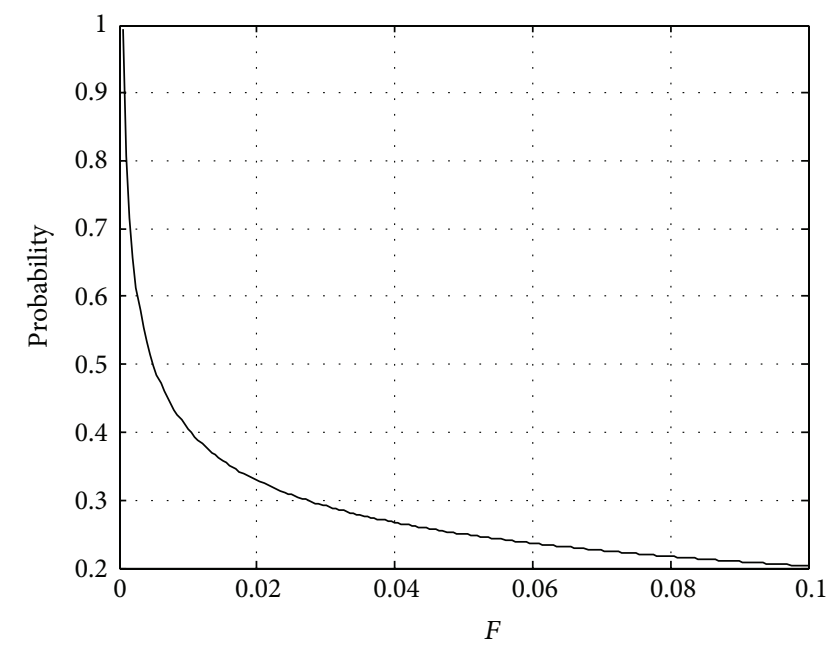

FIGURE 8: Probability of the slip versus the amplitude of the magma thrust strength for the parameters of Figure 7 with $e=0.9$ and $h=$ 0.1 .

curve giving the magnitude as a function of displacement [28].

To appreciate the influence of the external force on these two quantities, we draw each of them as a function of $F$. Figure 7 shows that the magnitude of the earthquake increases with the amplitude of the external force while the probability of an event decreases with the growth of $F$ (Figure 8). We could also note from these plots that, for small values of $F$, the appearing probability of the event is significant, but earthquakes which occur are not powerful since their magnitudes are very small.

\section{Discussions and Conclusion}

In this paper, we have examined the dynamics of a $1 D$ spring-block model for earthquakes subjected to cumulative 
strengths due to the motion of the tectonic plates and the upflow of magma during volcanism. The presence of the horizontal spring during the modeling has allowed for the introduction of a new parameter called the sound velocity. Through the direct integration method, we have explained the impacts of the magma thrust strength (amplitude $F$, frequency $\omega$ ) on the dynamics of the earthquakes. Indeed, we have shown that the displacement of the block is oscillatory and its amplitude increases with the increment of $F$ and the sound velocity when $\omega \neq 0$. We have also demonstrated that when the external frequency $\omega$ equals the pseudofrequency of the block's oscillation, the slip which occurs during this periodic movement is a stick-slip motion instead of a creep motion like that previously established [1]. It has appeared from our analytical investigations that the magma thrust strength deletes the first order phase transition observed in [1]. We have also noted that the power of the earthquake increases with the amplitude of the magma load while the corresponding event probability decreases with it.

The model studied in this paper represents a generalization of previous models about the behavior of a springblock model subjected to the strengths due to the relative motion between two plates since it takes into account the flow of magma during volcano emission. Based on the physical interpretations arising from this study on the mechanisms of the earthquakes' dynamics under magma thrust strengths, we hope the results established within this paper could be also valuable as an interesting application that can be used to illustrate this sort of concepts to graduate students.

In solving (17), we have limited investigations to the resonance case in which the frequency of the magma upflow equals the fundamental frequency of the spring block. In the corresponding nonresonance case, this equation cannot be solved analytically. Numerical integration of this relation is under consideration.

\section{Conflict of Interests}

There is no conflict of interests concerning the work done in this paper.

\section{References}

[1] G. L. Vasconcelos, "First-order phase transition in a model for earthquakes," Physical Review Letters, vol. 76, no. 25, pp. 48654868, 1996.

[2] R. Montagne and G. L. Vasconcelos, "Complex dynamics in a one-block model for earthquakes," Physica A: Statistical Mechanics and its Applications, vol. 342, no. 1-2, pp. 178-185, 2004.

[3] B. Erickson, B. Birnir, and D. Lavallée, "A model for aperiodicity in earthquakes," Nonlinear Processes in Geophysics, vol. 15, no. 1, pp. 1-12, 2008.

[4] R. Burridge and L. Knopoff, "Model and theoretical seismity," Bulletin of the Seismological Society of America, vol. 57, pp. 341371, 1967.

[5] G. L. Vasconcelos, M. de Sousa Vieira, and S. R. Nagel, "Phase transitions in a spring-block model of earthquakes," Physica A:
Statistical Mechanics and Its Applications, vol. 191, no. 1-4, pp. 69-74, 1992.

[6] M. De Sousa Vieira, G. L. Vasconcelos, and S. R. Nagel, "Dynamics of spring-block models: tuning to criticality," Physical Review E, vol. 47, no. 4, pp. R2221-R2224, 1993.

[7] P. G. Akishin, M. V. Altaisky, I. Antoniou, A. D. Budnik, and V. V. Ivanov, "Burridge-Knopoff model and self-similarity," Chaos, Solitons \& Fractals, vol. 11, no. 1-3, pp. 207-222, 2000.

[8] E. Brittany, Bjorn, and D. Lavallée, Periodicity, Chaos and Localisation in Burridge-Knopoff Model of an Earthquake with Dieterich-Ruina Friction, Center for Complex and Nonlinear Science, Santa Barbara, Calif, USA, 2010.

[9] S. A. Fedotov, "Magma rates in feeding conduits of different volcanic centres," Journal of Volcanology and Geothermal Research, vol. 9, no. 4, pp. 379-394, 1981.

[10] B. A. Chouet, "Excitation of a buried magmatic pipe: a seismic source model for volcanic tremor," Journal of Geophysical Research, vol. 90, no. 2, pp. 1881-1893, 1985.

[11] Y. Ida and M. Kumazawa, "Ascent of magma in a deformable vent," Journal of Geophysical Research, vol. 91, no. B9, pp. 92979301, 1986.

[12] B. A. Chouet, "A seismic model for the source of long period events and harmonic tremor," in Volcanic Seismology, P. Gasparini, R. Scarpa, and K. Aki, Eds., vol. 3 of IAVCEI Proceedings in Volcanology, pp. 133-156, Springer, 1992.

[13] Y. Ida, "Cyclic fluid effusion accompanied by pressure change: implication for volcanic eruptions and tremor," Geophysical Research Letters, vol. 23, no. 12, pp. 1457-1460, 1996.

[14] M. Kwékam, J.-P. Liégeois, E. Njonfang, P. Affaton, G. Hartmann, and F. Tchoua, "Nature, origin and significance of the Fomopéa Pan-African high-K calc-alkaline plutonic complex in the Central African fold belt (Cameroon)," Journal of African Earth Sciences, vol. 57, no. 1-2, pp. 79-95, 2010.

[15] C. H. Scholz, The Mechanics of Earthquakes and Faulting, Cambridge University Press, New York, NY, USA, 1990.

[16] P. Hähner and Y. Drossinos, "Nonlinear dynamics of a continuous spring-block model of earthquake faults," Journal of Physics A: Mathematical and General, vol. 31, no. 10, pp. L185-L191, 1998.

[17] J. S. Langer and C. Tang, "Rupture propagation in a model of an earthquake fault," Physical Review Letters, vol. 67, no. 8, pp. 1043-1046, 1991.

[18] H. Kanamori and D. L. Anderson, "Theoretical basis of some empirical relations in seismology," Bulletin of the Seismological Society of America, vol. 65, pp. 1073-1095, 1975.

[19] T. C. Hanks and H. Kanamori, "A moment magnitude scale," Journal of Geophysical Research B: Solid Earth, vol. 84, no. 5, pp. 2348-2350, 1979.

[20] H. Kanamori, H.-K. Thio, D. Dreger, E. Hauksson, and T. Heaton, "Initial investigation of the Landers, California, earthquake of 28 June 1992 using TERRAscope," Geophysical Research Letters, vol. 19, no. 22, pp. 2267-2270, 1992.

[21] T. Utsu, "Relationships between magnitude scales," in International Handbook of Earthquake, Engineering Seismology, W. H. K. Lee, H. Kanamori, P. C. Jennings, and C. Kisslinger, Eds., vol. 81-A of International Geophysics, pp. 733-746, Academic Press, Division of Elsevier, 2002.

[22] C. F. Richter, "An instrumental earthquake magnitude scale," Bulletin of the Seismological Society of America, vol. 25, pp. 132, 1935.

[23] B. Gutenberg, "Amplitudes of surface waves and magnitude of shallow earthquakes," Bulletin of the Seismological Society of America, vol. 35, pp. 3-12, 1945. 
[24] B. Gutenberg and C. F. Richter, "Earthquake magnitude, intensity, energy, and acceleration," Bulletin of the Seismological Society of America, vol. 46, pp. 105-146, 1956.

[25] A. V. Vvendenskaya, "The determination of displacement fields by means of dislocation theory," Izvestiya Akademii Nauk SSSR, Seriya Geograficheskaya, pp. 227-284, 1956.

[26] A. Keiti, "Estimation of earthquakes moment, released energy and stress-strain drop from wave spectrum," Bulletin of the Earthquake Research Institute, vol. 44, pp. 23-88, 1966.

[27] M. W. Dongmo, L. Y. Kagho, F. B. Pelap, G. B. Tanekou, Y. L. Makenne, and A. Fomethe, "Water effects on the first-order transition in a model of earthquakes," ISRN Geophysics, vol. 2014, Article ID 160378, 7 pages, 2014.

[28] P. Pascal, Qu'est-ce qui fait trembler la terre? EDP Science, 2003. 

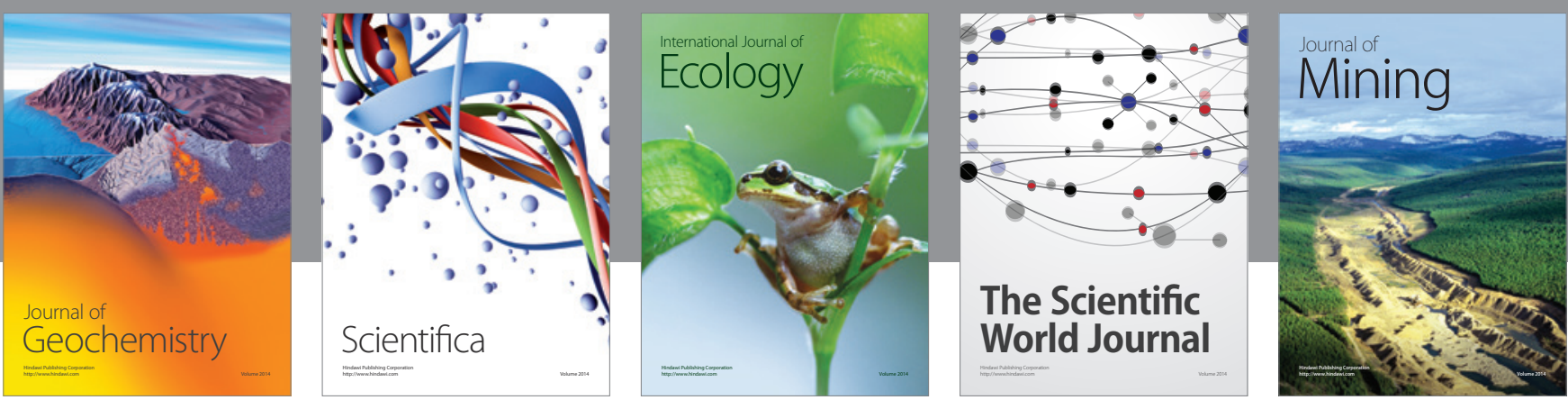

The Scientific World Journal
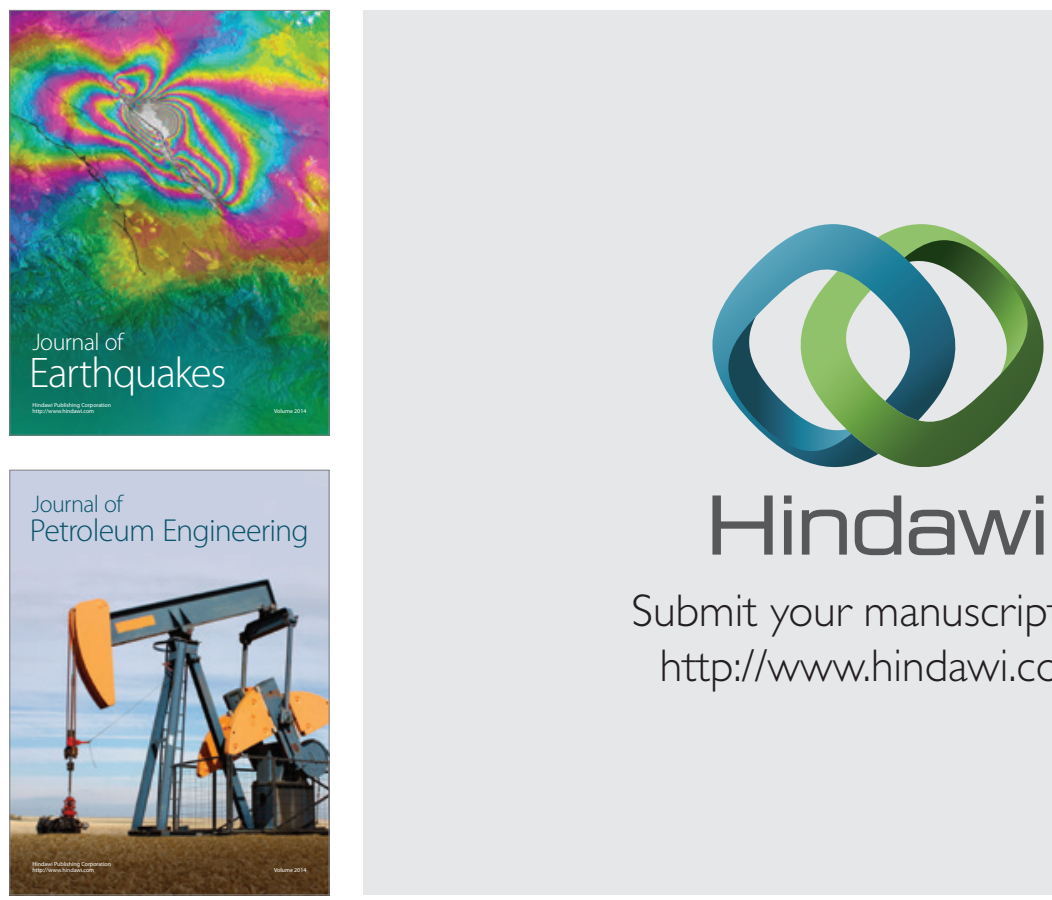

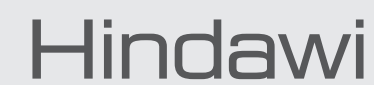

Submit your manuscripts at

http://www.hindawi.com
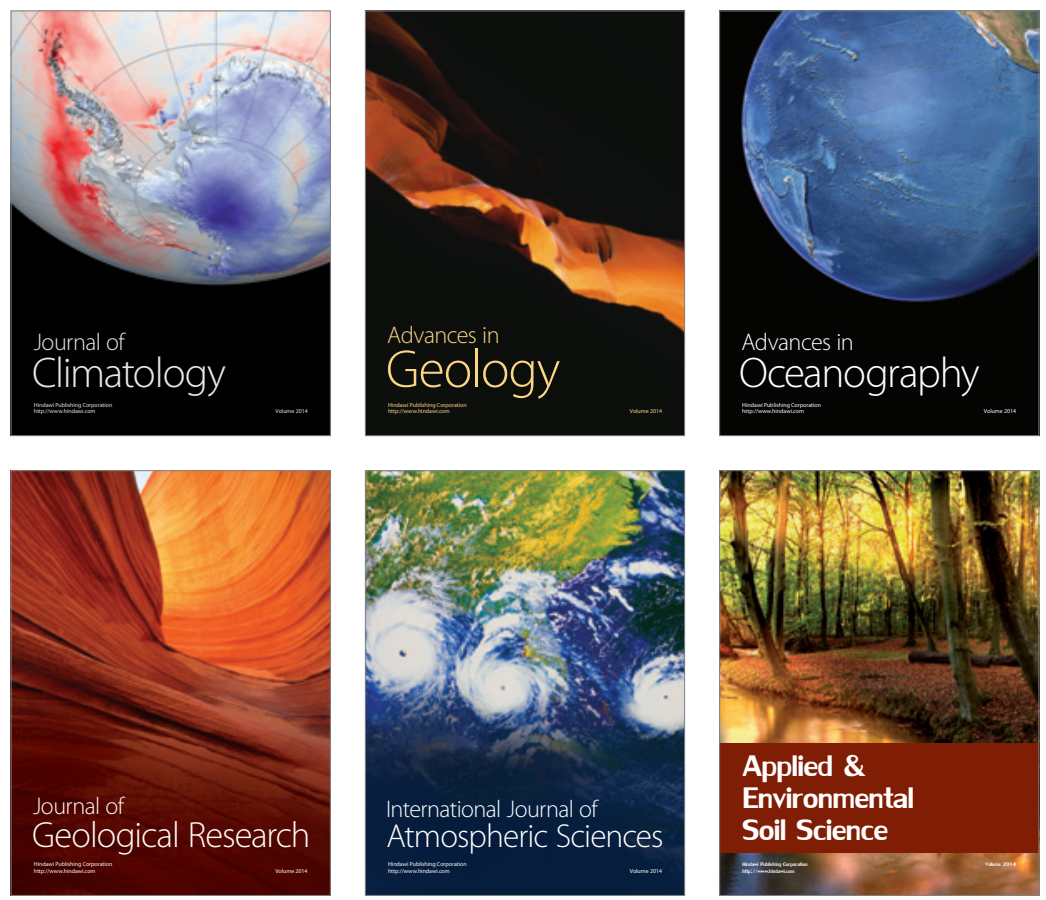
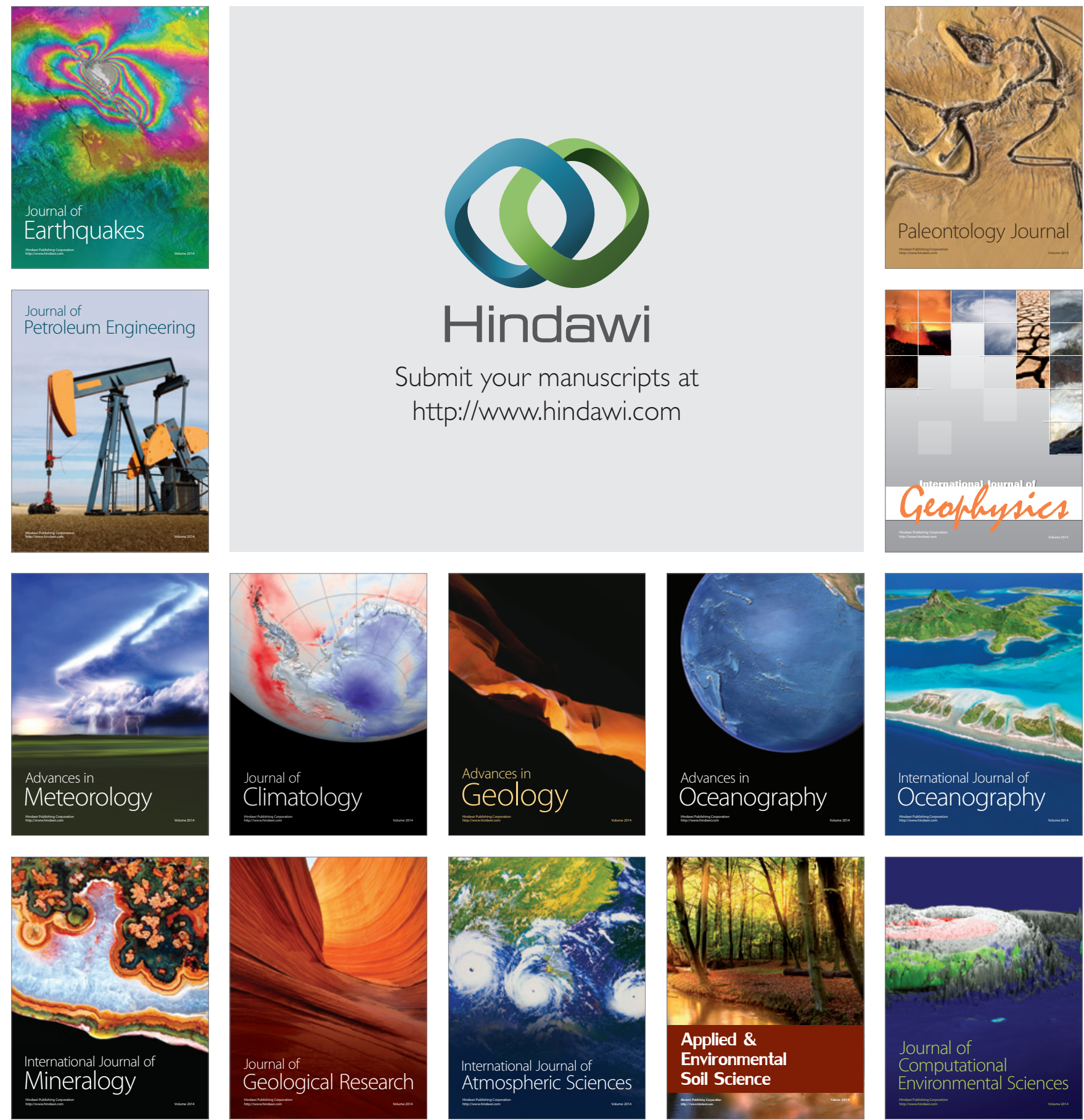